\title{
Die Relevanz von Global Health für die Schweiz
}

Nicolaus Lorenz, Bettina Borisch, Michaela Told, John-Paul Vader

1 Nach Basch P E. Textbook of International Health. Oxford Univ Pr.1999.

Korrespondenz: Nicolaus Lorenz executive MBA, MPH, MD Swiss Tropical and Public Health Institute Socinstrasse 59 Postfach

CH-4002 Basel

Tel. 0612848125

Fax 0612848103

nicolaus.lorenz@unibas.ch
Global Health ist nicht etwa nur ein neumodischer Begriff, sondern von wirklicher Bedeutung für die Schweiz. Von der Schweiz aus gesehen, scheint «Global Health» ein ferner und exotischer Ort zu sein: schwierig zu erreichen, eigenartig anziehend, vielleicht etwas gefährlich, aber mit der Aussicht, ein wertvolles und lohnendes Ziel zu sein [1]. Dieser Aspekt passt gut in das schweizerische Umfeld, da Herr und Frau Schweizer zu den Vielfliegern dieser Welt gehören. Reisen ist bereichernd, denn es bietet die Möglichkeit, fremde Kulturen kennenzulernen, und öffnet den Blick für Neues. In der klinischen Medizin sind wir gewohnt, über die Schweizer Grenzen hinauszuschauen und vom medizinischen Wissen aus anderen Teilen der Welt zu profitieren - auch wenn es manchmal etwas Zeit braucht, bis dieses Wissen Eingang in die hiesige klinische Praxis findet.

Für die «Public Health Schweiz» war und ist die globale Dimension von zentraler Bedeutung. Auch wenn die H1N1-Pandemie nicht die Auswirkungen hatte, die manche vorausgesagt haben, ist sie doch ein gutes Beispiel dafür, wie schnell sich eine lokale Epidemie zu einer Pandemie entwickeln kann.

Antworten auf die Probleme eines Gesundheitssystems müssen landesspezifisch gefunden werden. Aber sicherlich kann die Schweiz von Fehlern, aber auch Erfolgen anderer Ländern lernen - und umgekehrt. Beispielsweise haben die schweizerischen HIV-/ AIDS-Präventionskampagnen einen ausgezeichneten Ruf und viele Kampagnen in anderen Ländern beeinflusst.

Der Einfluss von schweizerischem Public HealthWissen zeigt sich auch darin, dass es in der Schweiz 18 WHO Collaborating Centres gibt (http://apps.who. int/whocc/). Dies sind Exzellenzzentren mit dem Auftrag, die Weltgesundheitsorganisation in verschiedenen technischen Bereichen zu unterstützen. Im Vergleich zu Deutschland mit 33 Zentren verfügt die Schweiz also über mehr als halb so viele Zentren und hat nur vier weniger als das benachbarte Frankreich (22). Die Schweiz verfügt zudem über Einrichtungen, etwa das Graduate Institute in Genf, die sich mit globaler Gesundheitsdiplomatie beschäftigen und in Europa einzigartig sind. In Genf, der Weltgesundheitshauptstadt, sind neben der Weltgesundheitsorganisation zahlreiche andere globale Gesundheitsinitiativen angesiedelt. Schweizerische Organisationen und Institutionen, wie z. B. das Schweizerische Tropen- und Public Health-Institut, arbeiten sehr eng mit dem Global
Fund to Fight HIV/AIDS und der Drugs for Neglected Diseases Initiative (DNDi) zusammen. Public Health Schweiz und das Institut für Sozial- und Präventivmedizin der Universität Genf beherbergen das Sekretariat der World Federation of Public Health Associations in Genf.

\section{Die Schweiz kann sicher von Feh- lern, aber auch Erfolgen anderer}

\section{Länder lernen}

Um die Global Health-Erfahrung in der Schweiz zu bündeln und internationale Erfahrungen einbringen zu können, wurde die Fachgruppe «Global Health» in der Fachgesellschaft «Public Health Schweiz» neu belebt.

Im vergangenen Jahr wurde der Versuch unternommen, eine für die Schweiz angepasste Arbeitsdefinition von Global Health zu erarbeiten. Auf der Basis einer umfassenden Sichtung der Literatur und eingehender Diskussionen, möchten wir folgende Definition vorschlagen: Global Health ist ein Gebiet für Forschung, Praxis und Policy, bei dem besonderer Wert gelegt wird auf die Verbesserung von Gesundheit im allgemeinen und auf den gerechten Zugang der Menschen zur Gesundheit im besonderen. Global Health beschäftigt sich mit transnationalen Gesundheitsfragen, den Determinanten und Lösungsansätzen. Viele Fachdisziplinen, nicht nur aus dem Gesundheitssektor, sind hierfür notwendig; die interdisziplinäre Zusammenarbeit wird gefördert und Global Health fördert die gute Governance, die es ermöglicht, sich abzeichnende Veränderungen im Umfeld frühzeitig zu erkennen und darauf rechtzeitig zu reagieren.

In Zukunft soll eine Plattform geschaffen werden, um internationale Erfahrungen in die Schweiz einbringen zu können und die schweizerischen Erfahrungen mit internationalen Partnern auszutauschen, etwa mit der European Public Health Association und der World Federation of Public Health Associations.

Last but not least wird sich die Fachgruppe hier in der Schweiz für das oben skizzierte breite Verständnis von Global Health einsetzen. Interessierte Fachpersonen sind eingeladen, hieran mitzuarbeiten (www.public-health.ch/). 